\title{
Utilisation de cinétiques du premier ordre en vue de caractériser la minéralisation de quel- ques sols agricoles
}

\author{
Jean-Emmanuel DELPHIN \\ avec la collaboration technique de Christian Bohonos et Christiane Huck \\ I.N.R.A., Station d'Agronomie, 28, rue de Herrlisheim, F 68021 Colmar
}

RÉSUMÉ

\begin{abstract}
Seize échantillons de sols agricoles provenant d'un réseau d'expérimentation sur la France ont été mis en incubation pendant $340 \mathrm{j}$ à $20^{\circ} \mathrm{C}$ et $80 \mathrm{p} .100$ d'humidité équivalente. Les mesures de dégagement cumulé de $\mathrm{CO}_{2}$ sont ajustées à 2 cinétiques du premier ordre :

$-\mathrm{Cm}=\mathrm{C}(1-\exp (-\mathrm{kt}))$ sur un intervalle de 0-340 j (cinétique 1) ou de 50-340 j (cinétique 1 bis),

- $\mathrm{Cm}=\mathrm{C}_{1}\left(1-\exp \left(-\mathrm{k}_{1} \mathrm{t}\right)\right)+\mathrm{C}_{2}\left(1-\exp \left(-\mathrm{k}_{2} \mathrm{t}\right)\right)$ sur un intervalle de 0-340 $\mathrm{j}$ (cinétique 2 ).

On suit la variation dans le temps des paramètres sur les 100 derniers jours d'incubation. La variabilité de $C$ et $k$ est plus élevée pour la cinétique 1 que 1 bis, avec parfois des écarts entre les valeurs obtenues. Les paramètres $\mathrm{C}_{2}$ et $\mathrm{k}_{2}$ présentent un palier plus ou moins large dont la valeur correspond en général au $C$ et $\mathrm{k}$ moyen de la cinétique 1 bis. Quel que soit le modèle utilisé, le choix des paramètres dépend de l'échelle de temps à laquelle on envisage la simulation. La liaison statistique entre $\mathrm{k}_{2}$ et les teneurs en argiles des sols est faible, $\mathrm{C}_{2}$ est plus étroitement corrélé au couple matières organiques-argiles. La combinaison de ces 2 paramètres $\left(C_{200}=C_{2}\left(1-\exp \left(-200 k_{2}\right)\right)\right.$ est fortement corrélée au rapport MO/argiles.
\end{abstract}

Mots clés additionnels : Incubation, respirométrie, $\mathrm{CO}_{2}$.

A mineralization study was conducted with 16 surface soils from field experiment stations in France. The soil samples were incubated at $20^{\circ} \mathrm{C}$ over 340 days. The cumulative $\mathrm{C}-\mathrm{CO}_{2}$ production was fitted to two firstorder kinetics :

$-\mathrm{Cm}=\mathrm{C}(1-\exp (-\mathrm{kt}))$ on a 0-340 days incubation (kinetic 1$)$ or on a 50-340 days incubation (kinetic 1 bis),

$-\mathrm{Cm}=\mathrm{C}_{1}\left(1-\exp \left(-\mathrm{k}_{1} \mathrm{t}\right)\right)+\mathrm{C}_{2}\left(1-\exp \left(-\mathrm{k}_{2} \mathrm{t}\right)\right)$ on a 0-340 days incubation (kinetic 2$)$.

The variation of the kinetic parameters with time was studied over the last 100 incubation days. The variability of $\mathrm{C}$ and $\mathrm{k}$ was higher with kinetic $\mathrm{I}$ than $\mathrm{I}$ bis and some values were different. Parameters $\mathrm{C}_{2}$ and $\mathrm{k}_{2}$ of values of kinetic 2 had stage values generally similar to the mean $\mathrm{C}$ or $\mathrm{k}$ over the last 100 days of kinetic 1 bis. The use of these kinetic parameters in mineralization models will depend on the time range of simulation. $k_{2}$ was poorly correlated with soil clay content, the correlation between $C_{2}$ and OM-clay was better. A combination of $C_{2}$ and $k_{2}\left(C_{200}=C_{2}\left(1-\exp \left(-200 k_{2}\right)\right)\right.$ was closely related to the ratio $O M /$ clay.

Additional key words : Incubation, respirometry, $\mathrm{CO}_{2}$.

\section{INTRODUCTION}

La mesure du dégagement du gaz carbonique est une méthode permettant de juger de l'activité microbienne globale d'un sol. Dans le cas de la minéralisation endogène, le dégagement du $\mathrm{CO}_{2}$ est étroitement lié à la libération d'azote minéral. La fourniture d'azote par cette voie peut satisfaire jusqu'à 50 p. 100 des besoins des grandes cultures. La rationalisation de la fertilisa- tion azotée nécessite une bonne connaissance des quantités produites et de leur libération au cours du temps. Cela suppose la maîtrise des cinétiques de minéralisation, en particulier l'élaboration de modèles de référence sur lesquels on peut faire agir les principaux facteurs du milieu.

De nombreuses études ont porté ces dernières années sur ce point, surtout en vue de définir le potentiel d'azote minéralisable No des sols (STANFORD \& 
TABLEAU 1

Caractéristiques physico-chimiques des sols (p. 1000 ).

Characteristics of the soils.

\begin{tabular}{|c|c|c|c|c|c|c|c|c|}
\hline $\mathrm{N}^{\circ}$ & $\begin{array}{l}\mathrm{pH} \\
\text { eau }\end{array}$ & $\begin{array}{l}\text { Arg. grossières* } \\
0,2-2 \mu \mathrm{m}\end{array}$ & Argiles* & Limons* & Sables* & $\begin{array}{l}\text { Calcaire } \\
\text { total }\end{array}$ & $\begin{array}{l}\text { Carbone } \\
\text { organique }\end{array}$ & $\mathbf{N}$ total \\
\hline 1 & 6,8 & 98 & 194 & 615 & 191 & - & 13,2 & 1,33 \\
\hline 2 & 6,3 & 81 & 127 & 451 & 421 & - & 7,4 & 0,84 \\
\hline 3 & 5,4 & 97 & 191 & 694 & 115 & - & 9,0 & 1,17 \\
\hline 4 & 6,7 & 121 & 245 & 574 & 178 & - & 12,5 & 1,30 \\
\hline 5 & 8,1 & 123 & 247 & 400 & 352 & 6 & 9,2 & 1,32 \\
\hline 7 & 7,3 & 97 & 190 & 765 & 53 & - & 14,5 & 1,61 \\
\hline 8 & 8,0 & 172 & 379 & 568 & 16 & 38 & 28,3 & 3,10 \\
\hline 9 & 8,3 & 150 & 254 & 324 & 246 & 174 & 12,7 & 1,34 \\
\hline 10 & 6,5 & 120 & 179 & 288 & 532 & - & 30,3 & 3,15 \\
\hline 11 & 6,8 & 129 & 235 & 476 & 290 & - & 8,8 & 1,03 \\
\hline 12 & 6,4 & 110 & 187 & 743 & 69 & - & 9,9 & 1,01 \\
\hline 13 & 6,2 & 109 & 151 & 455 & 394 & - & 35,0 & 2,88 \\
\hline 14 & 8,1 & 117 & 155 & 119 & 183 & 535 & 12,7 & 1,37 \\
\hline 16 & 6,2 & 91 & 129 & 732 & 138 & - & 15,2 & 1,20 \\
\hline
\end{tabular}

* après décarbonatation pour les sols calcaires.

SMITH, 1972 ; MARY \& REMY, 1979 ; DEANS et al., 1986). Nous avons cherché ici, par des mesures de $\mathrm{CO}_{2}$, à préciser les possibilités d'utilisation des cinétiques du premier ordre, décrivant au mieux la minéralisation de quelques sols agricoles et à en estimer les paramètres, en vue d'élaborer des modèles de simulation de la minéralisation endogène. Nous avons également examiné les relations entre les paramètres de ces cinétiques et les caractéristiques des sols étudiés afin de disposer de moyens d'estimation plus rapides que les méthodes d'incubation.

\section{MATÉRIEL ET MÉTHODES}

\section{A. Sols étudiés}

Les échantillons proviennent d'un réseau français d'essais de fertilisation du maîs conduits par l'AGPM sur des parcelles d'agriculteurs. Le choix des sols visait une forte diversité des caractères physico-chimiques, en particulier au niveau des teneurs en matières organiques, en argiles et en calcaire (tabl. 1).

Ces essais concernent des rotations céréalières avec un précédent blé ou orge, les pailles étant enfouies ( $\mathrm{n}^{\mathrm{os}} 2$, $11,14,15)$ ou exportées $\left(n^{o s} 1,4\right)$ ou maïs avec restitution des cannes $\left(\mathrm{n}^{\circ} 5,6,7,9,10,16\right)$ ou sans restitution $\left(n^{\circ s} 3,8,13\right)$. Des amendements organiques sous forme de fumier (30-60 t) ont été effectués dans certains cas, 2 à 6 mois avant le prélèvement $\left(\mathrm{n}^{\mathrm{O}} 1,7,8\right.$, 15, 16). La parcelle $n^{\circ} 12$ comporte un précédent lupin enfoui à la sortie de l'hiver.

Les échantillons ont été prélevés dans les 20 premiers centimètres de la couche arable au cours du printemps 1986. Ils ont été conservés sans autre traitement à $5{ }^{\circ} \mathrm{C}$, pendant 1 à $15 \mathrm{j}$, jusqu'au début de l'incubation.

\section{B. Incubation}

Les essais d'incubation sont conduits à $20^{\circ} \mathrm{C}$ sur la terre échantillonnée (comportant environ $1 \mathrm{~kg}$ de terre sèche) en pots cylindriques de $0,01 \mathrm{~m}^{2}$ de section, la densité apparente variant de 1 à 1,2 . L'humidité est maintenue à 80 p. 100 de l'humidité équivalente par des apports réguliers d'eau. Le $\mathrm{CO}_{2}$ est mesuré après piégeage, pendant 1 à $5 \mathrm{j}$ selon l'avancement de l'incubation, dans des coupelles contenant une solution de soude $N / 2$. Entre 2 mesures, chaque pot est recouvert d'un sachet plastique de 21 en présence de $30 \mathrm{ml}$ de soude $\mathrm{N}$. On détermine le $\mathrm{CO}_{2}$ piégé par dosage de la soude non carbonatée en présence de thymolphtaléine après précipitation des carbonates par du chlorure de baryum. L'expérience est conduite, selon les cas, avec 2 à 6 répétitions en début d'expérience. Les valeurs cumulées du dégagement de $\mathrm{CO}_{2}$ sont calculées en admettant une variation linéaire entre 2 points de mesure et après élimination des données s'écartant de plus de 5 p. 100 de la tendance générale. Le calcul du $\mathrm{CO}_{2}$ dégagé est effectué à partir du $25^{\mathrm{e}} \mathrm{j}$ d'incubation afin de minimiser les répercussions d'une extraminéralisation possible en début d'expérience. L'ajustement aux cinétiques du premier ordre est obtenu par la méthode des moindres carrés non linéaires (NASH, 1979).

\section{Méthodes analytiques}

Les méthodes utilisées ont été les suivantes :

- azote total : méthode Kjeldahl,

- carbone organique : analyseur de carbone après calcination (servocoulomètre Eraly),

- calcaire : mesure de $\mathrm{CO}_{2}$ sur analyseur de carbone (servocoulomètre) après attaque acide,

- fractionnements granulométriques : décantation pour les fractions les plus grossières et centrifugation pour les autres $(<2 \mu \mathrm{m})$ après décarbonatation, selon les cas, et destruction des matières organiques par le peroxyde d'hydrogène. 


\section{RÉSULTATS ET DISCUSSION}

\section{A. Ajustement à des cinétiques du premier ordre}

Nous avons dans un premier temps ajusté les valeurs cumulées de $\mathrm{CO}_{2}$ à une cinétique du premier ordre du type :

$$
\mathrm{Cm}=\mathrm{C}(1-\exp (-\mathrm{kt}))
$$

$\mathrm{Cm}$ représente la quantité de carbone minéralisé $(\mathrm{mg} \mathrm{C}$ $\mathrm{kg}^{-1}$ ), C l'asymptote de la courbe (potentiel minéralisable théorique, $\mathrm{mg} \mathrm{C} \mathrm{kg}^{-1}$ ) et $\mathrm{k}$ la constante de vitesse $\left(\mathrm{j}^{-1}\right)$.

Les valeurs des paramètres estimés varient d'un rapport 1 à 3 selon les sols (tabl. 2), avec des coefficients de détermination très élevés $(0,95-0,98)$. Les résidus ( $\mathrm{Cm}$ observé - $\mathrm{Cm}$ estimé) ne sont cependant pas indépendants de la durée d'incubation, ce qui laisse supposer une liaison entre les paramètres et le temps. Si l'on réalise les ajustements en supprimant progressivement les points des 100 derniers jours d'incubation, on peut en effet remarquer que les valeurs estimées des paramètres ne sont pas stables : $\mathrm{C}$ augmente avec la durée d'incubation alors que $\mathrm{k}$ diminue (fig. 1). Le fait d'écarter les 50 premiers jours d'incubation des points ajustés (cinétique 1 bis) réduit en général la variabilité des paramètres et donne souvent des valeurs nettement différentes (tabl. 2) ; dans certains cas pourtant $\left(\mathrm{n}^{\circ \mathrm{os}} 5\right.$, 13) la variabilité demeure élevée. L'amélioration des ajustements obtenus en éliminant les données provenant du tout début d'incubation a déjà été mentionnée dans les études de No (STANFORD et al., 1974 ; MARY \& RÉMY, 1979). On réduit ainsi les effets de débris organiques facilement minéralisables et ceux de l'extraminéralisation consécutive à la destruction partielle de la biomasse microbienne lors du conditionnement des échantillons de sol avant l'expérience.

TABLEAU 2

Valeur des paramètres selon la cinétique d'ajustement utilisée.

Estimates of the parameters according to the fitting kinetics used.

\begin{tabular}{|c|c|c|c|c|c|c|c|c|c|c|}
\hline \multirow{3}{*}{$\mathrm{N}^{\circ}$} & \multirow{2}{*}{\multicolumn{8}{|c|}{$\begin{array}{c}\text { Cinétique } 1 \\
\text { moyennes (coefficient de variation } 1 \text { bis } \\
\text { sur les } 100 \text { derniers jours d'incubation }\end{array}$}} & \multirow{2}{*}{\multicolumn{2}{|c|}{ Cinétique 2}} \\
\hline & & & & & & & & & & \\
\hline & \multicolumn{2}{|c|}{$\mathrm{C}\left(\mathrm{mg} \mathrm{C} \mathrm{kg}^{-1}\right)$} & \multicolumn{2}{|c|}{$k \times 10^{3}\left(\mathrm{j}^{-1}\right)$} & \multicolumn{2}{|c|}{ C } & \multicolumn{2}{|r|}{ k } & $\begin{array}{l}\mathrm{C}_{2} \text { minimum } \\
(\mathrm{mg} C \mathrm{~kg}\end{array}$ & $\begin{array}{l}k_{2} \operatorname{maximum} \\
\times 10^{-3}\left(j^{-1}\right)\end{array}$ \\
\hline 1 & 1040 & $(3,4)$ & 5,2 & $(5,0)$ & 1140 & $(1,7)$ & 3,9 & $(3,0)$ & 1110 & 4,1 \\
\hline 2 & 830 & $(1,4)$ & 4,4 & $(2,0)$ & 910 & $(2,4)$ & 3,5 & $(3,9)$ & 890 & 3,7 \\
\hline 3 & 1850 & $(9,5)$ & 2,6 & $(11,5)$ & 3220 & $(4,1)$ & 1,2 & $(4,9)$ & 2950 & 1,3 \\
\hline 4 & 1000 & $(6,7)$ & 3,8 & $(8,4)$ & 1380 & $(3,4)$ & 2,1 & $(4,4)$ & 1530 & 1,8 \\
\hline 5 & 1050 & $(10,8)$ & 3,5 & $(13,2)$ & 1580 & $(15,7)$ & 1,9 & $(18,5)$ & 900 & 3,9 \\
\hline 6 & 1530 & $(8,0)$ & 3,3 & $(10,5)$ & 1920 & $(4,8)$ & 2,3 & $(6,6)$ & 1960 & 2,1 \\
\hline 7 & 1515 & $(5,0)$ & 3,4 & $(6,6)$ & 1900 & $(2,7)$ & 2,3 & $(3,5)$ & 1840 & 1,8 \\
\hline 8 & 1640 & $(15,9)$ & 3,9 & $(18,7)$ & 4650 & $(2,5)$ & 0,9 & $(2,8)$ & $5000^{*}$ & $0,9^{*}$ \\
\hline 9 & 1260 & $(8,4)$ & 3,2 & $(11,3)$ & 2190 & $(4,6)$ & 1,4 & $(5,7)$ & 2120 & 1,4 \\
\hline 10 & 1690 & $(4,1)$ & 3,4 & $(5,4)$ & 1970 & $(2,3)$ & 2,6 & $(3,3)$ & 1940 & 2,6 \\
\hline 11 & 920 & $(3,9)$ & 4,7 & $(5,6)$ & 1030 & $(1,6)$ & 3,5 & $(2,6)$ & 1030 & 3,4 \\
\hline 12 & 1050 & $(2,7)$ & 6,2 & $(4,7)$ & 1070 & $(2,8)$ & 5,1 & $(6,3)$ & 1240 & 1,9 \\
\hline 13 & 1880 & $(9,2)$ & 4,2 & $(11,8)$ & 2270 & $(15,6)$ & 3,0 & $(20,0)$ & 1760 & 4,3 \\
\hline 14 & 1390 & $(3,5)$ & 3,3 & $(4,5)$ & 1435 & $(5,5)$ & 3,1 & $(9,0)$ & 1400 & 3,2 \\
\hline 15 & 750 & $(4,1)$ & 7,4 & $(6,3)$ & 840 & $(0,8)$ & 5,0 & $(1,6)$ & 850 & 4,6 \\
\hline 16 & 1030 & $(5,7)$ & 3,9 & $(7,9)$ & 1150 & $(5,4)$ & 3,1 & $(7,9)$ & 1110 & 3,8 \\
\hline
\end{tabular}

* valeur obtenue par extrapolation.
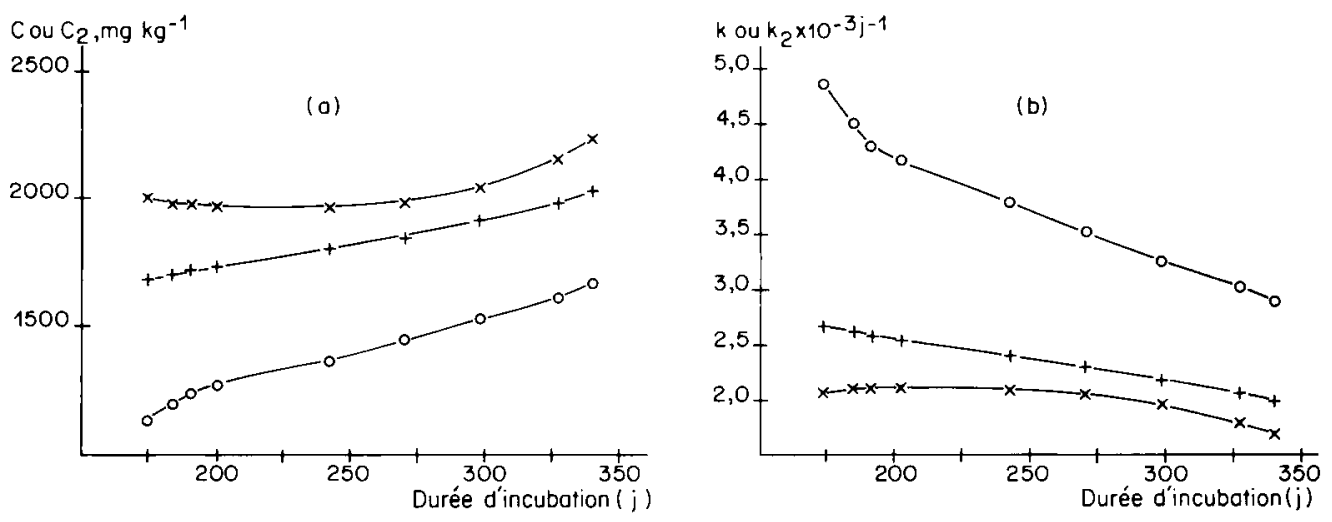

Figure 1

Variation des paramètres dans le temps (sol $\left.n^{\circ} 6\right)$ c cinétique 1 , + cinétique $I$ bis, x cinétique 2.

Parameter change in relation to incubation time (soil $\left.n^{\circ} 6\right) \circ$ kinetic 1 , + kinetic 1 bis, $x$ kinetic 2 
On peut également ajuster la totalité des données à une double cinétique du premier ordre, ce qui permet de prendre en compte les 2 phases de la minéralisation :

$$
\mathrm{Cm}=\mathrm{C}_{1}\left(1-\exp -\left(\mathrm{k}_{1} \mathrm{t}\right)\right)+\mathrm{C}_{2}\left(1-\exp \left(-\mathrm{k}_{2} \mathrm{t}\right)\right)
$$

où $\mathrm{C}_{1}$ et $\mathrm{k}_{1}$ sont les paramètres associés à la phase rapide, $\mathrm{C}_{2}$ et $\mathrm{k}_{2}$ à la phase lente.

Cette méthode, déjà utilisée (NUSKE \& RICHTER, 1981 ; DEANS et al., 1986), permet théoriquement de séparer la composante explosive de la minéralisation et la phase plus stable qui nous intéresse. Le coefficient de détermination est amélioré (supérieur à 0,997), mais la variabilité des paramètres après retrait progressif des derniers points n'est pas réduite. Les paramètres présentent un palier minimal $\left(\mathrm{C}_{2}\right)$ ou maximal $\left(\mathrm{k}_{2}\right)$ en général bien marqué (fig. 1), sauf pour le sol $n^{\circ} 12$, et qui intervient sur un laps de temps correspondant approximativement à la demi-vie $\left(\log (2) / k_{2}\right)$ du terme plus lent, avec une variabilité souvent élevée en dehors $\mathrm{du}$ palier. Ces valeurs concordent avec $\mathrm{C}$ et $\mathrm{k}$ moyens obtenus par ajustement à une cinétique 1 bis (tabl. 2), mis à part le sol $n^{\circ} 12$ qui a reçu un apport organique rẹcent et les sols présentant une variabilité élevée de $\mathrm{C}$ et $\mathrm{k}$ ( $\mathrm{n}^{\text {os }} 5$ et 13). Cependant, la valeur des critères $\mathrm{k}_{2}$ maximal et $C_{2}$ minimal utilisée ici n'est appréciée qu'empiriquement, la validité de cette méthode reste à démontrer. Les paramètres du premier terme ont un comportement identique à $\mathrm{C}_{2}$ et $\mathrm{k}_{2}$. Les valeurs moyennes minimales de $C_{1}$ et maximales de $k_{1}$ (respectivement de $40 \pm 20 \mathrm{mg} \mathrm{kg}^{-1}$ et de $0,005 \pm 0,0025 \mathrm{j}^{-1}$ ) peuvent être associées à un faible pool organique facilement minéralisé et dont la taille est liée - bien que de manière lâche - aux apports organiques récents (fumier et pailles de maïs) ; seul le sol $n^{\circ} 12$ se distingue nettement : $C_{1}=360 \mathrm{mg} \mathrm{kg}^{-1}$ et $\mathrm{k}_{1}=0,013 \mathrm{j}^{-1}$. La dérive dans le temps des paramètres et leur instabilité tiennent au fait que les modèles d'ajustement, malgré des $\mathbf{R}^{2}$ très élevés, ne sont qu'une représentation sommaire des processus étudiés et que $\mathrm{C}$ et $\mathrm{k}$ des cinétiques 1 et 1 bis d'une part, et les paramètres des 2 termes de la cinétique 2 d'autre part, sont interdépendants.

Du fait de leur variabilité dans le temps, on peut difficilement envisager d'extrapoler les valeurs des paramètres obtenues dans des conditions déterminées. Dans la perspective d'une utilisation au champ, une durée d'incubation à $20^{\circ} \mathrm{C}$ d'environ 1 an paraît nécessaire pour obtenir une estimation adéquate des paramètres $\mathrm{C}$ et $\mathrm{k}$ de minéralisation. Le recours à une cinétique double se justifie surtout dans le cas de sols présentant une forte activité en début d'incubation, de plus, il n'est valable que si les paramètres sont définis sur un laps de temps correspondant à l'échelle d'utilisation.

\section{B. Estimation des paramètres de minéralisation}

Nous avons cherché des moyens simples d'appréciation des paramètres de ces cinétiques reposant sur leurs relations statistiques avec certaines caractéristiques des sols. Pour l'estimation de $\mathrm{C}$ (ou de $\mathrm{C}_{2}$ ) nous avons considéré que $20 \mathrm{p} .100 \mathrm{du} \mathrm{CO}_{2}$ produit par les sols calcaires provenait de la décarbonatation biologique. $\mathrm{Ce}$ chiffre rend compte de l'acidification consécutive à la nitrification (FAURIE, 1977) mais aussi de la production d'acides organiques microbiens (TARDIEUX \& TARDIEUX, 1975). Il s'agit d'une approximation, la nature des carbonates (ROBERT et al., 1980) et vraisemblablement leur taille devant également intervenir.

L'analyse statistique porte sur $\mathrm{C}_{2}$ et $\mathrm{k}_{2}$. On dispose de peu d'études sur les relations entre les paramètres des cinétiques et les caractéristiques des sols. Les corrélations négatives entre $\mathrm{k}_{2}$ et les teneurs en argiles sont assez lâches (tabl. 3). Des relations du même type peuvent être tirées d'études de No (STANFORD \& SMITH, 1972 ; JUMA et al., 1984). Bien que les résultats présentés ici ne portent pas sur la même échelle de temps, ils concordent avec les liaisons observées entre les argiles et le coefficient de minéralisation apparent des essais de longue durée au champ (REMY \& MARINLAFLÈCHE, 1974). Le faible effectif de sols calcaires ne permet pas de dégager de liaisons entre $\mathrm{k}_{2}$ et le taux de calcaire.

TABLEAU 3

Régression entre les paramètres de la cinétique 2 et les caractéristiques physico-chimiques des sols.

Regression between the parameters of kinetic 2 and some characteristics of the soils.

\begin{tabular}{|c|c|c|c|}
\hline & Equations de régression & $\mathrm{R}^{2}$ & $\mathrm{~F}$ \\
\hline $\mathrm{k}_{2}$ & $=-0,008$ Arg. tot. $+4,5$ & 0,28 & $5,5^{*}$ \\
\hline $\mathrm{C}_{2}$ & $=50 \mathrm{C} \mathrm{org} .+840$ & 0,25 & $4,6^{*}$ \\
\hline $\mathrm{C}_{2}$ & $=47 \mathrm{C}$ org. $+4,4$ Arg. tot. -50 & 0,43 & $5,0^{*}$ \\
\hline $\mathrm{C}_{2}$ & $=0,26 \mathrm{C}$ org. $\times$ Arg. tot. +730 & 0,57 & $18,8^{* *}$ \\
\hline $\mathrm{C}_{200}$ & $=15,5$ C org. +340 & 0,70 & $32,8 * *$ \\
\hline $\mathrm{C}_{200}$ & $=15,8 \mathrm{C}$ org. $-0,38$ Arg. tot. +420 & 0,74 & $18,6^{* *}$ \\
\hline $\mathrm{C}_{200}$ & $=16,0 \mathrm{C}$ org. $-0,65 \mathrm{Arg}$. gros. +415 & 0,73 & $17,7^{* *}$ \\
\hline$C_{200}$ & $=2480 \mathrm{C}$ org. $/$ Arg. tot. +385 & 0,74 & $38,6^{* *}$ \\
\hline$C_{200}$ & $=1915 \mathrm{C}$ org $/$ Arg. gros +330 & 0,77 & $47,5^{* *}$ \\
\hline
\end{tabular}

$*$ significatif seuil $<5$ p. 100.

** significatif seuil $<1$ p. 100 .

$k_{2} \quad:$ constante de vitesse cinétique $2\left(10^{-3} \times \mathrm{j}^{-1}\right)$.

$\mathrm{C}_{2} \quad: \mathrm{C}$ potentiellement minéralisable cinétique 2

$\mathrm{C}_{200} \quad$ : Carbone minéralisé sur 200 j calculé selon 3

$\left(\mathrm{mg} \mathrm{C} \mathrm{kg}^{-1}\right)$.

C org. : Carbone organique (p. 1000$)$

Arg. tot. : Argiles $<2 \mu \mathrm{m}$ (p. 1000$)$

Arg. gros. : Argiles $0,2-2 \mu \mathrm{m}$ (p. 1000$)$

Pour $\mathrm{C}_{2}$, les liaisons statistiques sont un peu plus étroites, surtout avec le modèle multiplicatif (tabl. 3). Les teneurs en argiles et en matières organiques sont corrélées positivement au carbone potentiellement minéralisable déterminé par ajustement. Les relations matières organiques- $\mathrm{C}_{2}$ sont comparables à celles obtenues pour No (STANFORD \& SMITH, 1972). Ces régressions vont dans le sens des résultats de CHAUSSOD et al. (1986) montrant que les argiles contribuent à augmenter le stock organique minéralisable tout en limitant sa biodégradabilité, ce qui peut constituer une explication des corrélations positives de $C_{2}$ à la fois avec les matières organiques et les argiles.

L'estimation de $\mathrm{k}_{2}$ à partir des régressions dégagées ici est trop imprécise pour être utilisée dans la simulation de la minéralisation endogène des sols. De plus, le fait de déterminer séparément des paramètres qui sont 
autocorrélés peut encore accrô̂tre le risque d'erreurs sur le calcul de la minéralisation ; des analyses statistiques complémentaires ont donc porté sur une combinaison des 2 paramètres:

$$
\mathrm{C}_{200}=\mathrm{C}_{2}\left(1-\exp \left(-200 \mathrm{k}_{2}\right)\right)
$$

$C_{200}$ est la minéralisation endogène théorique sur $200 \mathrm{j}$, $200 \mathrm{j}$ constituant un intervalle de temps compatible avec l'échelle à laquelle les simulations peuvent être envisagées dans une application au champ.

Comme le pouvoir minéralisateur des sols (DELPHIN, 1986), $C_{200}$ est étroitement lié aux teneurs en matières organiques et en argiles, avec un effet plus marqué des argiles grossières dans le cas du modèle $\mathrm{C}$ organique/argiles (tabl. 3).

Le calcul de $\mathrm{k}_{2}$ est obtenu à partir de l'équation (3), $\mathrm{C}_{2}$ et $\mathrm{C}_{200}$ étant tirés d'une des régressions figurant au tableau 3. Cette estimation de $C_{2}$ et $k_{2}$ a l'avantage de tenir compte de la dépendance des 2 paramètres. L'utilisation d'une cinétique du premier ordre avec ces paramètres n'est cependant valable que pour une échelle de temps cadrant avec celle de leur détermination expérimentale. La validité des résultats de simulation repose en grande partie sur la précision de l'estimation statistique de $\mathrm{C}_{200}$. Si globalement cette estimation est satisfaisante, certains points ( $\mathrm{n}^{\mathrm{os}} 3,6$ et 12$)$ se singularisent (fig. 2).

\section{CONCLUSION}

Les cinétiques du premier ordre utilisées pour le calcul de No ont été appliquées ici à l'ajustement de mesures de dégagement de $\mathrm{CO}_{2}$ de sols agricoles mis en incubation en laboratoire. Les paramètres ainsi établis ne sont pas stables dans le temps. Une des causes de leur variabilité tient à l'extraminéralisation et à la présence de résidus organiques facilement minéralisables ; elle peut être réduite soit en éliminant les premiers points de mesure (cinétique $1 \mathrm{bis}$ ), soit en utilisant une double cinétique (2). Ces 2 méthodes donnent des valeurs de paramètres en général proches, cependant, quelle que soit la méthode adoptée, leur estimation reste dépendante de l'intervalle de temps sur lequel porte l'ajustement. De ce fait, le choix des valeurs des paramètres pour une simulation dépendra de l'échelle

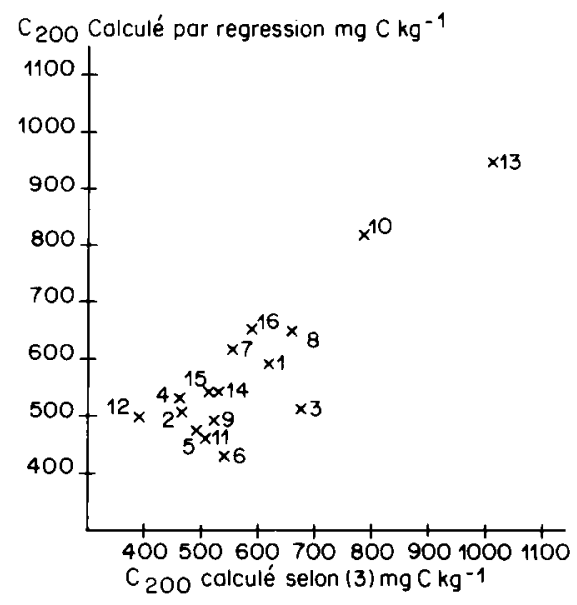

Figure 2

Relation entre $C_{200}$ calculé selon (3) et par la régression $C_{200}=$ $1915 \mathrm{Clot} / \mathrm{arg}$. gros. +330.

Relation between $C_{200}$ given by (3) and estimated according to $C_{200}=1915$ Ctot/arg. gros. +330.

de temps à laquelle on travaille. Les liaisons statistiques plus ou moins étroites des paramètres avec certaines caractéristiques des sols telles que teneur en matières organiques et en argiles constituent un moyen de les estimer, simple et satisfaisant quant à la précision.

La mesure du $\mathrm{CO}_{2}$ comme indicateur de minéralisation est plus aisée et plus rapide que celle de l'azote minéral, surtout dans les études cinétiques qui nécessitent un grand nombre de déterminations. Les résultats obtenus ici constituent une première étape. La conversion du $\mathrm{CO}_{2}$ produit en azote minéralisé net est un point important à résoudre ultérieurement, si l'on veut appliquer ces cinétiques à une estimation plus fine de la minéralisation endogène des sols agricoles.

Recu le 28 septembre 1987. Accepté le 23 février 1988.

\section{REMERCIEMENTS}

Cette étude a été menée avec l'aide de l'Association Générale des Producteurs de Maïs (AGPM).

\section{RÉFÉRENCES BIBLIOGRAPHIQUES}

Chaussod R., Nicolardot B., Catroux O., Chrétien J., 1986. Relations entre les caractéristiques physico-chimiques et microbiologiques de quelques sols cultivés. Bull. Ass. Fr. Etud. Sol Sci. Sol, 2 , 213-226.

Deans J. R., Molina J. A. E., Clapp C. E., 1986. Models for predicting potentially mineralizable nitrogen and decomposition rate constant. Soil Sci. Soc. Am. J., 50, 323-326.

Delphin J. E., 1986. Evaluation du pouvoir minéralisateur de sols agricoles en fonction de leurs caractéristiques physico-chimiques. Agronomie, 6 (5), 453-458.

Faurie G., 1977. Etude in vitro du rôle de la nitrification sur la lixiviation du calcium dans les sols calcaires. Bull. Ass. Fr. Etud. Sol Sci. Sol., 4, 207-218.
Juma N. G., Paul E. A., Mary B., 1984. Kinetic analysis of net nitrogen mineralization in soil. Soil Sci. Soc. Am. J., 48, 753-757.

Mary B., Rémy J. C., 1979. Essai d'appréciation de la capacité de minéralisation de l'azote des sols de grande culture. 1. Signification des cinétiques de minéralisation de la matière organique humifiée. Ann. agron., 30 (6), 513-527.

Nash J. C., 1979. Compact numerical methods for computers: linear algebra and function minimisation. Adam Hilger, Bristol, $227 \mathrm{p}$.

Nuske A., Richter J., 1981. N-mineralization in loessparbrownearths : incubation experiments. Plant Soil, 59, 237-247.

Rémy J. C., Marin-Laflèche A., 1974. L'analyse de terre : réalisation d'un programme d'interprétation automatique. Ann. agron., 25 (4), 607-632. 
Robert M., Veneau G., Berrier J., 1980. Action des composés organiques sur la dissolution des carbonates. Bull. Ass. Fr. Etud. Sol Sci. Sol, 3, 231-245.

Stanford G., Smith S. J., 1972. Nitrogen mineralization potentials of soils. Soil Sci. Soc. Am. Proc., 36, 465-472.
Stanford G., Carter J. N., Smith S. J., 1974. Estimate of potentially mineralizable soil nitrogen based on short-term incubations. Soil Sci. Soc. Am. Proc., 38, 99-102.

Tardieux A., Tardieux P., 1975. Solubilisation d'éléments minéraux dans la rhizosphère. Soc. Bot. Fr., Coll. Rhizosphère, 139-144. 